\title{
Surface Tuning on Metal Organic Frameworks for Film Bulk Acoustic Resonator Sensors Array
}

\author{
X. Yan ${ }^{1,2}$, H. Qu*1,2, W. Pang ${ }^{\star 1,2}$, H. Zhang ${ }^{1,2}$, X. Duan ${ }^{\star 1,2}$ \\ 1 State key Laboratory of Precision Measuring Technology \& Instruments, Tianjin University, China \\ ${ }^{2}$ College of Precision instrument \& Opto-electronics Engineering, Tianjin University, China \\ e-mail: hemi.qu@tju.edu.cn,weipang@tju.edu.cn, xduan@tju.edu.cn.
}

\begin{abstract}
:
In this paper, an in-situ fabrication method of coating metal organic framework (MOF) on film bulk acoustic resonator (FBAR) was described. This method is mild and straightforward, able to produce dense and homogeneous sorptive layers on FBAR. The surface of MOF layer on FBAR was further modified with several types of self-assembled monolayers (SAMs). The introduction of SAMs on MOF can vary the response of FBAR sensors toward volatile organic compounds (VOCs). The prepared 4 FBAR sensors were arrayed to facilitate the recognition of VOCs. The response of FBAR sensors array toward methanol, ethanol, n-propanol and hexane, was analyzed by pattern recognition algorithm, which led to a clear separation of the VOCs.
\end{abstract}

Key words: Film Bulk Acoustic Wave Resonator, Self- Assembled Monolayer, Metal Organic Framework, Sensor Array, Volatile Organic Compounds

\section{Introduction}

The last decade has witnessed rapid emergence of a variety of micro gas sensors including acoustic wave sensors, metal oxide semiconducting sensors, capacitive sensors, field effect transistor sensors et al. Recently, film bulk acoustic resonators (FBAR) are gaining increasing attention because of their small size, light weight, low cost and mass sensitive characteristics. FBAR is a typical MEMS piezoelectrical device like quartz crystal microbalance (QCM) where thin piezoelectrical layer is sandwiched between top and bottom electrodes. Being different with QCM which only resonates at frequencies limited to a few tens of $\mathrm{MHz}, \mathrm{FBAR}$ operates at frequencies extended to the $\mathrm{GHz}$ range. Such high operating frequency can significantly improve the performance of piezoelectrical mass sensors because the mass sensitivity of piezoelectrical mass sensors increases with the square of operating frequency [1].

The choice of sorptive material as sensitive layer on the FBAR sensor is critical in improving sensing performance because the interaction of sorptive layer with vapors directly controls the sensor's sensitivity and selectivity. In order to improve sensor performance, a variety of materials on FBAR have been employed, including Cu2+/11-mercaptoundecanoic acid, antibody, monolayers [2]. However, achieving sufficient selectivity in those sensors is still challenging because of the existence of nonspecific dispersion interactions at the gassolid interface [3]. Sensor arrays in combination with statistical analysis could be employed to overcome this challenge by mimicking biological olfaction systems comprising multiple receptors and neuronal pattern recognition. In this contribution, a metal organic framework (MOF) material, namely HKUST-1, was employed as sorptive layer on the FBAR sensor. In order to produce sensors array, several types of self-assembled monolayer were introduced on HKUST-1. In the end, the response of FBAR sensors array with several VOCs was studied.

\section{Results}

FBARs were fabricated in our lab by standard MEMS fabrication process. The coating of HKUST-1 on passivation layer (AIN) of FBAR was realized in two steps with Wöll's method (Figure $1 \mathrm{~A}(\mathrm{a}-\mathrm{b}))$. The growth of self-assembled monolayers on HKUST-1 was achieved firstly by coating PDMS and then by chemical vapor deposition (Figure $1 \mathrm{~A}(\mathrm{c}-\mathrm{d})$ ). The structure of FBARs was characterized with scanning 
electron microscope (SEM) (Figure 1B). The step-by-step growth of HKUST-1 on FBARs was confirmed by monitoring frequency changing during layer-by-layer deposition of HKUST-1 (Figure 1C). The response of sensors array with four volatile organic compounds (VOCs), such as methanol, ethanol, n-propanol and hexane, was analyzed by pattern recognition algorithm, which led to a clear separation of the VOCs (Figure 2).

\section{References}

[1] H. Zhang, E. S. Kim, Micromachined Acoustic Resonant Mass Sensor, Journal of

(A)
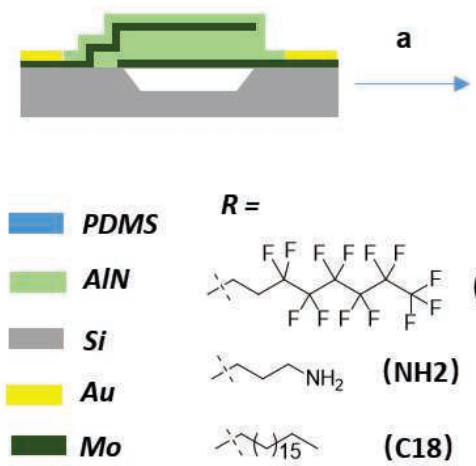

(B)

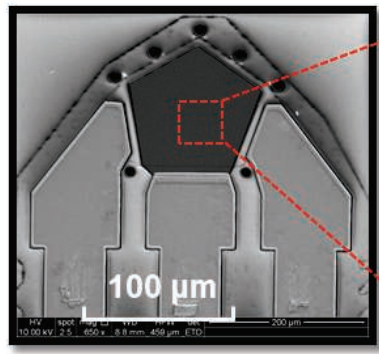

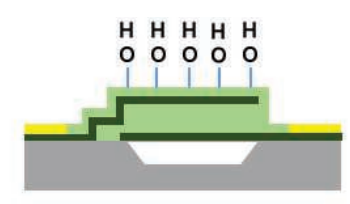

$\begin{array}{lllll}0 & \mathrm{O} & \mathrm{O} & \mathrm{O} & \mathrm{O}\end{array}$

R $\quad \mathbf{R}$

(F)

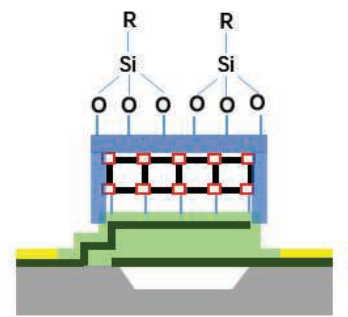

R@PDMS@HKUST b

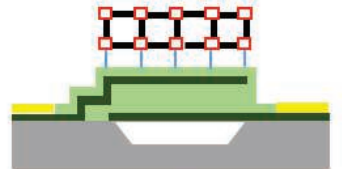

c

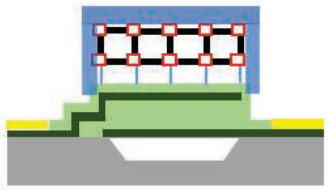

PDMS@HKUST

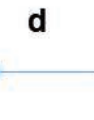

(C)

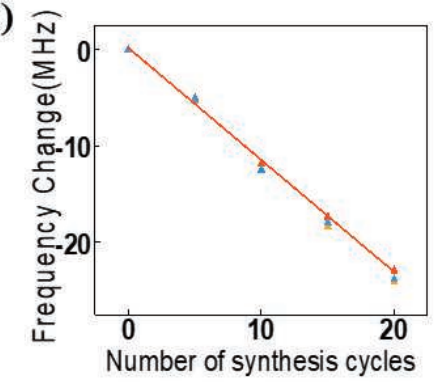

Fig.1. (A) Fabrication process of sorptive coating on FBAR sensors: a) plasma activation of FBAR; b) dipping FBAR repeatedly in $\mathrm{Cu}(\mathrm{OAc})_{2}$ solution, $\mathrm{H}_{3} B T C$ solution and ethanol for several cycles; $\left.c\right)$ heating FBAR in presence of PDMS stamps in a vacuum oven at $235^{\circ} \mathrm{C}$; d) plasma and chemical vapor deposition of APTES, OTES and PFDTS. (B) SEM image of FBAR sensor (left) and HKUST-1 film on FBAR (right). (C) Frequency shift during fabrication of HKUST-1 by repeatedly dipping as in $b$.
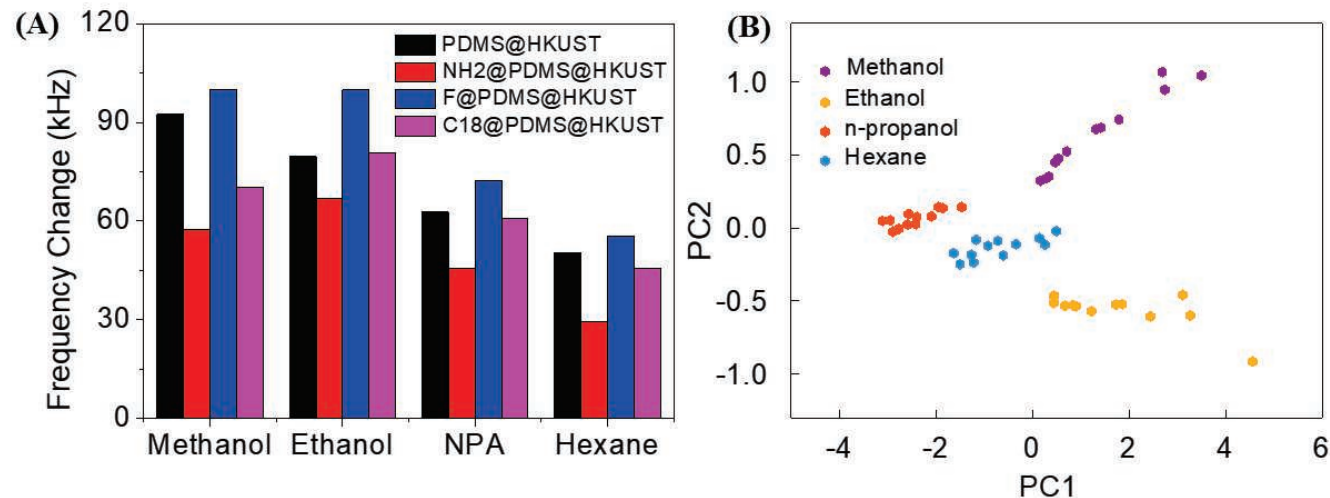

Figure 2. (A) Frequency changes of FBAR sensor array upon exposure to $100 \mathrm{ppm}$ volatile organic compounds at room temperature; (B) Principal component score plots of an array of 4 FBAR sensors array to 4 VOCs. 